\title{
HYPERSURFACES WHOSE TANGENT GEODESICS DO NOT COVER THE AMBIENT SPACE
}

\author{
SÉRGIO MENDONÇA AND HEUDSON MIRANDOLA
}

(Communicated by Jon G. Wolfson)

We dedicate this work to our beloved wives Cristina and Fabiola

\begin{abstract}
Let $x: \Sigma^{n} \rightarrow M^{n+1}$ be an immersion of an $n$-dimensional connected manifold $\Sigma$ in an $(n+1)$-dimensional connected complete Riemannian manifold $M$ without conjugate points. Assume that the union of geodesics tangent to $x$ does not cover $M$. Under these hypotheses we have two results. The first one states that $M$ is simply connected provided that the universal covering of $\Sigma$ is compact. The second result says that if $x$ is a proper embedding and $M$ is simply connected, then $x(\Sigma)$ is a normal graph over an open subset of a geodesic sphere. Furthermore, there exists an open star-shaped set $A \subset M$ such that $\bar{A}$ is a manifold with the boundary $x(\Sigma)$.
\end{abstract}

\section{INTRODUCTION}

Let $x: \Sigma^{n} \rightarrow M^{n+1}$ be an immersion of a connected $n$-dimensional manifold $\Sigma$ in a connected complete $(n+1)$-dimensional Riemannian manifold $M$. A very strong condition would be to assume that $x$ is totally geodesic. Here we will make a much weaker assumption, namely, that the union of geodesics tangent to $x$ does not cover $M$. More precisely, let $W=W(x)$ be the set of points of $M$ that do not lie on any geodesic tangent to $x$. We could ask in what situations we could have $W \neq \emptyset$. The first important answer to this question was given by Halpern [Hp]. To state it we first recall a well-known definition.

Definition 1.1. A subset $B$ of a complete manifold $M$ is said to be star-shaped with respect to $x_{0}$ if $x_{0} \in B$ and for any point $p \in B$ there exists a unique minimal normal geodesic joining $x_{0}$ and $p$, and the image of that unique geodesic is contained in $B$.

Theorem A (Halpern [Hp]). Let $x: \Sigma^{n} \rightarrow \mathbf{R}^{n+1}, n \geq 2$, be an immersion of a closed manifold $\Sigma$ with $W \neq \emptyset$. Then, the following conclusions hold:

(1) $x$ is an embedding;

(2) $\Sigma^{n}$ is diffeomorphic to the Euclidean sphere;

(3) there exists a diffeomorphic disk $D$ with $\partial D=x(\Sigma)$; furthermore $D$ and its interior $\operatorname{int}(D)$ are star-shaped with respect to any point of $W$.

Received by the editors November 3, 2006.

2000 Mathematics Subject Classification. Primary 53C42; Secondary 53C22.

Key words and phrases. Riemannian manifold, hypersurface, geodesic, star-shaped set.

This work was partially supported by CNPq, Brasil. 
Beltagy [Be] extended this result to the case where $M$ is a complete and simply connected Riemannian manifold without conjugate points, using the fact that the exponential map is a diffeomorphism and applying Theorem A to the tangent space at any point of $W$. Alexander [Al] modified Theorem A, restricting the attention to the union $V$ of tangent spaces at saddle points of $\Sigma$, and obtained a weaker conclusion.

Remark 1. Theorem A and Alexander's Theorem can be extended to the case where $M$ is the standard sphere $S^{n+1}$ by using the stereographic projection associated with the antipodal point $-P$ where $P \in W$ in the case of Theorem A and $P \in S^{n+1}-V$ in the case of Alexander's Theorem.

Alencar and Frensel proved in $[\mathrm{AF}]$ that, if $M$ is a space form $Q^{n+1}(c)$ and $x$ is minimal with $W$ being a nonempty open set, then $x$ is totally geodesic. This result for a minimal immersion $x: M^{2} \rightarrow Q_{c}^{3}, c \geq 0$, with nonempty $W$ has been proved by Hasanis and Koutroufiotis $[\mathrm{HK}]$. Another result in $[\mathrm{AF}]$ says that if $\Sigma^{n} \subset Q^{n+1}(c)$ is closed and has constant mean curvature with $W \neq \emptyset$, then $\Sigma$ is a round sphere.

Now we state our first result.

Theorem 1.1. Let $x: \Sigma^{n} \rightarrow M^{n+1}, n \geq 2$, be an immersion, where $M$ is a connected complete Riemannian manifold without conjugate points and with $W \neq \emptyset$. If the universal covering of $\Sigma$ is compact, then $M$ is simply connected.

Remark 2. The inclusion $T^{n} \subset T^{n+1}$ of flat tori shows that the hypothesis that $\tilde{\Sigma}$ is compact is essential in Theorem 1.1.

The following result is new even in the case that $M=\mathbf{R}^{n+1}$. First we recall the definition of a normal graph.

Definition 1.2. Let $S, X$ be contained in a manifold $M$, where $S$ is an embedded submanifold. We say that $X$ is a normal graph over $S$ if there exists a homeomorphism $h: S \rightarrow X$ such that for any point $x \in S$, the image $h(x)$ is connected to $x$ by a unique minimal geodesic, and that unique geodesic is orthogonal to $S$.

Theorem 1.2. Let $\Sigma$ be a connected properly embedded hypersurface in a simply connected complete Riemannian manifold $M$ without conjugate points with $W \neq \emptyset$. Then we have:

(1) $\Sigma$ is a normal graph over an open subset of a geodesic sphere;

(2) there exists an open set $A$ such that $A$ and its closure $\bar{A}$ are star-shaped with respect to any point of $W$; furthermore, $\bar{A}$ is a manifold that has $\Sigma$ as its boundary.

Remark 3. If we consider the spiral $S$ given by $r=1+2^{-\theta}$ in polar coordinates, the product $S \times \mathbf{R}^{n-1} \subset \mathbf{R}^{n+1}$ shows that the condition that $\Sigma$ is properly embedded is essential in Theorem 1.2.

\section{Proof of Theorem 1.1}

Let $x: \Sigma^{n} \rightarrow M^{n+1}$ be an immersion where $M$ is a connected Riemannian manifold without conjugate points. Let $\pi: \widetilde{M} \rightarrow M$ be the universal covering of $M$ with the induced metric and let $\nu: \widetilde{\Sigma} \rightarrow \Sigma$ be the universal covering of $\Sigma$. By the Fundamental Lifting Theorem, for any $p \in \Sigma, \bar{p} \in \nu^{-1}(p)$ and $\tilde{q} \in \pi^{-1}(x(p))$, 
there exists an immersion $y: \widetilde{\Sigma} \rightarrow \widetilde{M}$, namely the lifting of $x$, satisfying $y(\bar{p})=\tilde{q}$ and such that the diagram below commutes:

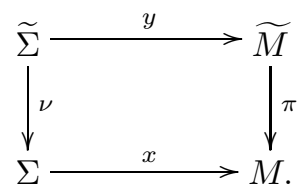

Let $W(y) \subset \widetilde{M}$ be the set of points that do not lie on any geodesic tangent to the immersion $y$. Let $\mathcal{A}$ be the set of liftings of $x$.

Claim 2.1. For $W=W(x)$ it follows that

$$
\pi^{-1}(W)=\bigcap_{y \in \mathcal{A}} W(y) .
$$

Proof. Assume that $\tilde{q} \notin \pi^{-1}(W)$. Since $q=\pi(\tilde{q}) \notin W$, there exists a geodesic $\gamma:[0, a] \rightarrow M$ with $\gamma(0)=q$ that is tangent to the immersion $x$ at $t=a$. The geodesic $\gamma$ can be lifted to a geodesic $\tilde{\gamma}:[0, a] \rightarrow \widetilde{M}$ with $\tilde{\gamma}(0)=\tilde{q}$ and such that $\tilde{\gamma}$ is tangent to some immersion $y \in \mathcal{A}$ at $t=a$. Thus $\tilde{q} \notin W(y)$.

Conversely, assume that $\tilde{q} \notin W(y)$ for some $y \in \mathcal{A}$. Then there exists a geodesic $\tilde{\gamma}$ tangent to the immersion $y$ containing $\tilde{q}$. The geodesic $\gamma:=\pi \circ \tilde{\gamma}$ is tangent to the immersion $x$ and contains $\pi(\tilde{q})$. Therefore $\pi(\tilde{q}) \notin W$; hence $\tilde{q} \notin \pi^{-1}(W)$.

To prove Theorem 1.1 we assume that $\widetilde{\Sigma}$ is compact. Take $x_{0} \in W$ and fix some lifting $y \in \mathcal{A}$. By Claim 2.1, $\pi^{-1}\left(x_{0}\right) \subset W(y)$. It follows from Beltagy's Theorem $[\mathrm{Be}]$ that $y(\widetilde{\Sigma})$ is an embedded sphere that is the boundary of a compact topological disk $D$ containing the set $\pi^{-1}\left(x_{0}\right)$. We also have that $D$ and its interior int(D) are star-shaped with respect to any point of $W(y)$, and thus they are star-shaped with respect to any point of $\pi^{-1}\left(x_{0}\right)$. Since $\pi^{-1}\left(x_{0}\right)$ is discrete and contained in a compact disk, the set $\pi^{-1}\left(x_{0}\right)$ and the group $\operatorname{Aut}(\pi)$ of automorphisms of $\pi$ are both finite. For any $\phi \in \operatorname{Aut}(\pi)$, we have that $\bar{y}:=\phi \circ y$ is also a lifting of $x$ since $\pi=\pi \circ \phi$.

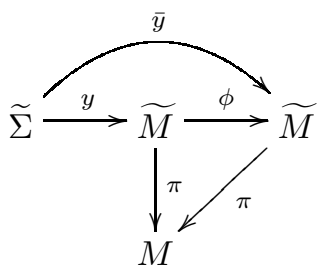

Again we have that $\pi^{-1}\left(x_{0}\right) \subset W(\bar{y})$ and that $\bar{y}(\widetilde{\Sigma})$ is embedded as the boundary of the compact topological disk $\phi(D)$ containing the set $\pi^{-1}\left(x_{0}\right)$. We also have that $\phi(D)$ and $\operatorname{int}(\phi(D))$ are star-shaped with respect to any point of $\pi^{-1}\left(x_{0}\right)$. Thus we have that

$$
E=\bigcap_{\phi \in \operatorname{Aut}(\pi)} \phi(D)
$$

is invariant under $\operatorname{Aut}(\pi)$ and that $\operatorname{int}(E)$ is star-shaped with respect to any point of $\pi^{-1}\left(x_{0}\right)$. In fact, since $\operatorname{Aut}(\pi)$ is finite, it is easy to see that $\operatorname{int}(E)=$ $\bigcap_{\phi \in \operatorname{Aut}(\pi)} \operatorname{int}(\phi(D))$. Thus $\operatorname{int}(E)$ is star-shaped with respect to any point in $\pi^{-1}\left(x_{0}\right)$, since it is the intersection of star-shaped sets. 
Claim 2.2. E is a compact topological disk.

Proof. Fix $\tilde{x}_{0} \in \pi^{-1}\left(x_{0}\right)$. We know that $\operatorname{int}(E)$ is star-shaped with respect to $\tilde{x}_{0}$. Fix a unit vector $v \in T_{\tilde{x}_{0}} \widetilde{M}$ and $\phi \in \operatorname{Aut}(\pi)$. Since $\tilde{x}_{0} \in W(\phi \circ y)$, we claim that the geodesic $\gamma: t \mapsto \exp _{\tilde{x}_{0}} t v$ meets transversely the hypersurface $\phi \circ y(\widetilde{\Sigma})=\partial \phi(D)$ at a unique point. In fact, there exists a first time $t_{v, \phi}$ such that $\gamma$ intersects $\partial \phi(D)$ since $\phi(D)$ is star-shaped with respect to $\tilde{x}_{0}$. Now we prove that this intersection is unique. Indeed, since $W(\phi \circ y) \neq \emptyset$, the geodesic $\gamma$ intersects $\partial \phi(D)$ transversely. Since $\phi(D)$ is a manifold with boundary $\partial \phi(D)$, we have that $\gamma(t) \notin \phi(D)$ for $t>t_{v, \phi}$ sufficiently close to $t_{v, \phi}$. If $\gamma$ intersects $\partial \phi(D)$ a second time this will contradict the fact that $\phi(D)$ is star-shaped with respect to $\tilde{x}_{0}$.

Since the intersection is a transversal at $t_{v, \phi}$, the time $t_{v, \phi}$ will depend smoothly on $v$. Let

$$
t(v)=\min _{\phi \in \operatorname{Aut}(\pi)} t_{v, \phi}
$$

Thus $t(v)$ depends continuously on $v$. Given $w \in E$, set

$$
v(w)=\frac{\left(\exp _{\tilde{x}_{0}}\right)^{-1} w}{\left\|\left(\exp _{\tilde{x}_{0}}\right)^{-1} w\right\|} .
$$

Let $B \subset T_{\tilde{x}_{0}} \widetilde{M}$ be the compact unit disk centered at 0 . Now we define $F: B \rightarrow E$ given by

$$
F(z)=\exp _{\tilde{x_{0}}} t(z /\|z\|) z,
$$

for $z \neq 0$, and $F(0)=\tilde{x}_{0}$. It is not difficult to see that $F$ has a continuous inverse $G: E \rightarrow B$ given by

$$
G(w)=\frac{1}{t(v(w))}\left(\exp _{\tilde{x}_{0}}\right)^{-1} w
$$

for $w \neq \tilde{x}_{0}$ and $G\left(\tilde{x}_{0}\right)=0$.

Finally, for any $\phi \in \operatorname{Aut}(\pi)$, we have that $\phi$ must have a fixed point in $E$, since $\phi(E) \subset E$, and $E$ is a compact disk by Claim 2.2. Thus we have that $\operatorname{Aut}(\pi)$ is trivial and $M$ is simply connected. Theorem 1.1 is proved.

\section{Proof of Theorem 1.2}

Let $M$ be a complete and simply connected Riemannian manifold without conjugate points. Let $\Sigma \subset M$ be a connected and properly embedded hypersurface satisfying $W \neq \emptyset$.

Fix $x_{0} \in W$. In particular $x_{0} \notin \Sigma$. Since $\Sigma$ is properly embedded there exists some small geodesic sphere $S$ centered at $x_{0}$ that does not intersect $\Sigma$. We define $F: \Sigma \rightarrow S$ as follows. Given $p \in \Sigma$, there exists a unique normal geodesic $\gamma:=$ $\left[x_{0}, p\right]$ joining $x_{0}$ and $p$. We know that $\gamma$ is orthogonal to $S$ at a unique intersection point, which we will call $F(p)$. Since $x_{0} \in W$ it follows that $\gamma$ is also transverse to $\Sigma$; hence $F: \Sigma \rightarrow S$ is a local diffeomorphism onto its open image $F(\Sigma) \subset S$. Thus, to show that $F: \Sigma \rightarrow F(\Sigma)$ is a diffeomorphism it is sufficient to show that $F$ is injective.

Define the set

$$
\mathcal{C}:=\left\{p \in \Sigma \mid \text { the cardinality } \#\left(\left[x_{0}, p\right] \cap \Sigma\right)=1\right\} .
$$

We need to prove that $\mathcal{C}=\Sigma$. 
Claim 3.1. $\mathcal{C} \neq \emptyset$. In fact, using that $\Sigma$ is a properly embedded hypersurface, we obtain that there exists a point $p_{0} \in \Sigma$, satisfying $d\left(x_{0}, p_{0}\right)=\min _{p \in \Sigma} d\left(x_{0}, p\right)$. Thus $p_{0} \in \mathcal{C}$.

Claim 3.2. $\Sigma-\mathcal{C}$ is open as a subset of $\Sigma$.

To prove this we take $x_{1} \in \Sigma-\mathcal{C}$. So there exists $x_{2} \in \Sigma$ with $x_{2} \neq x_{1}$ and $x_{2} \in\left[x_{0}, x_{1}\right]$. In particular we have $F\left(x_{1}\right)=F\left(x_{2}\right)=q$. Since $F$ is a local diffeomorphism, it is not difficult to see that there exist disjoint neighborhoods of $x_{1}$ and $x_{2}$ in $\Sigma$ mapped by $F$ onto the same neighborhood of $q \in S$. Thus we conclude that $\Sigma-\mathcal{C}$ is open in $\Sigma$.

Claim 3.3. $\Sigma-\mathcal{C}$ is closed as a subset of $\Sigma$.

In fact, take a sequence $x_{k} \rightarrow a \in \Sigma$, with $x_{k} \in \Sigma-\mathcal{C}$. Since $\Sigma$ is properly embedded, there exists an open neighborhood $U$ of $a \in M$ such that the intersection $\Sigma \cap U$ is connected and the restriction $\left.F\right|_{\Sigma \cap U}$ is a diffeomorphism onto its open image. By definition of $\mathcal{C}$, for each $k$ there exists a point $y_{k} \neq x_{k}$ with $y_{k} \in$ $\left[x_{0}, x_{k}\right] \cap \Sigma$. Since $\left(x_{k}\right)$ is bounded, we have that $\left(y_{k}\right)$ is also bounded. So we can assume by passing to a subsequence that $\left(y_{k}\right)$ converges to some point $b \in\left[x_{0}, a\right]$. Since $\Sigma$ is properly embedded we have that $b \in \Sigma$. Since $\left.F\right|_{\Sigma \cap U}$ is injective we have that $y_{k} \notin U$; hence $b \neq a$. So we conclude that $a \in \Sigma-\mathcal{C}$.

Thus $\mathcal{C}=\Sigma$ by connectedness of $\Sigma$ and $F: \Sigma \rightarrow F(\Sigma)$ is a diffeomorphism.

Now we will prove that $\Sigma$ is the boundary of an open star-shaped set with respect to $x_{0}$. Consider the set

$$
A:=\left\{z \in M \mid \#\left(\left[x_{0}, z\right] \cap \Sigma\right)=0\right\} .
$$

Given $z \in A$, the distance between $\left[x_{0}, z\right]$ and $\Sigma$ is positive, since $\Sigma$ is properly embedded. This implies that $A$ is open.

We state that $\bar{A}-A=\Sigma$. In fact, given $p \in \Sigma$, the geodesic segment $\left[x_{0}, p\right]-$ $\{p\} \subset A$; hence $\Sigma \subset \bar{A}$. Clearly, we have $A \cap \Sigma=\emptyset$; hence $\Sigma \subset \bar{A}-A$. Now take $p \in \bar{A}-A$. Assume by contradiction that $p \notin \Sigma$. Since $p \notin A$ the geodesic $\left[x_{0}, p\right]$ intersects $\Sigma$ transversely at a unique point $q \neq p$. Consider the unit vector $v \in$ $T_{x_{0}} M$ such that $\exp _{x_{0}} t_{0} v=p$ for some $t_{0}>0$. Since $F$ is a diffeomorphism onto its open image, there exists an open neighborhood $U$ of $v$ in the sphere $S^{n-1} \subset T_{x_{0}} M$ such that geodesic $t \mapsto \exp _{x_{0}} t w$ meets $\Sigma$ at a unique point for any $w \in U$. Since $\Sigma$ is properly embedded we can choose $U$ and $\epsilon>0$ sufficiently small such that $\exp _{x_{0}} t w \notin \Sigma$ if $\left|t-t_{0}\right|<\epsilon$. This defines a neighborhood $V$ of $p$ with $V \subset M-A$. This contradicts the fact that $p \in \bar{A}$.

Clearly we have that $A$ and $\bar{A}=A \cup \Sigma$ are star-shaped with respect to $x_{0}$. To conclude the proof we need to show that $\bar{A}$ is a manifold with boundary $\Sigma$. In fact, take a point $p \in \Sigma$, with $p=\exp _{x_{0}} t_{0} v$ for some unit vector $v \in T_{x_{0}} M$. Again there exists an open neighborhood $U$ of $v$ in $S^{n-1} \subset T_{x_{0}} M$ such that for each $w \in U$ the geodesic $t \mapsto \exp _{x_{0}} t w$ meets $\Sigma$ transversely at a unique point $q_{w}=\exp _{x_{0}} t_{w} w$. So the time $t_{w}$ depends smoothly on $w$. Thus a small neighborhood $W$ of $p$ in $\bar{A}$ can be defined as

$$
W=\exp _{x_{0}}\left\{t w \mid w \in U, t_{w}-\epsilon<t \leq t_{w}\right\},
$$

where $\epsilon>0$ is small enough. Thus we have proved that $\bar{A}$ is a smooth manifold with boundary $\Sigma$. 


\section{ACKNOWLEDGMENTS}

The authors thank Professor Manfredo do Carmo for useful discussions during the preparation of this paper.

\section{REFERENCES}

[AF] Alencar, H., Frensel, K., Hypersurfaces whose tangent geodesics omit a nonempty set, Differential Geometry, Pitman Monogr. Surveys Pure Appl. Math., 52, Longman Sci. Tech., Harlow, (1991), 1-13. MR1173029 (93f:53048)

[Al] Alexander, S., Saddle points of compact hypersurfaces. Geometriae Dedicata, 6 (1977), no.3, 353-362. MR0482547 (58:2613)

[Be] Beltagy, M., Immersions into manifolds without conjugate points, J. Inst. Math. Comput. Sci. Math. Ser. 3 (1990), no. 3, 265-271. MR1104375 (92f:53057)

[HK] Hasanis, T., Koutroufiotis, D. A property of complete minimal surfaces, Trans. Amer. Math. Soc., 281 (1984), no. 2, 833-843. MR722778 (85j:53064)

[Hp] Halpern, B., On the immersion of an $n$-dimensional manifold in $n+1$-dimensional Euclidean space, Proc. Amer. Math. Soc., 30 (1971), 181-184. MR0286116 (44:3330)

Departamento de Análise, Instituto de Matemática, Universidade Federal FlumiNense, Niterói, RJ, CEP 24020-140, Brasil

E-mail address: mendonca@mat.uff.br

E-mail address: sergiomendoncario@yahoo.com.br

Instituto de Matemática Pura e Aplicada, Rio de Janeiro, RJ, CEP 22460-320, Brasil Current address: Departamento de Engenharia e Ciências Exatas, Centro Universitário Norte do Espírito Santo, Universidade Federal do Espírito Santo, São Mateus, ES, CEP 29933-480, Brasil

E-mail address: heudson@impa.br 